\title{
Intraoperative, rapid administration of high-dose fentanyl by a dysfunctioning plunger lock of a syringe pump; the siphon effect
}

\author{
Sung-Hoon Kim, and Gyu-Sam Hwang \\ Department of Anesthesiology and Pain Medicine, University of Ulsan College of Medicine, Asan Medical Center, Seoul, Korea
}

Syringe pumps are designed to deliver drugs in a safe manner, however, these devices can be potentially dangerous if operated improperly. A 50-year-old male was scheduled to undergo an elective liver transplantation. After induction of anesthesia, the Baxter AS40A pump was loaded with a 50 $\mathrm{ml}$ Becton Dickinson syringe containing 2,500 $\mu \mathrm{g}$ of fentanyl. Continuous infusion at $5 \mathrm{ml} / \mathrm{hr}$ was started without knowing that part of the plunger lock, i.e. the upper plunger clamp assembly, was damaged (Fig. 1), and, therefore, the plunger was incompletely clamped without notice. Ten minutes later the patient experienced marked sinus bradycardia and hypotension for which a rescue drug of $10 \mu \mathrm{g}$ of epinephrine was injected
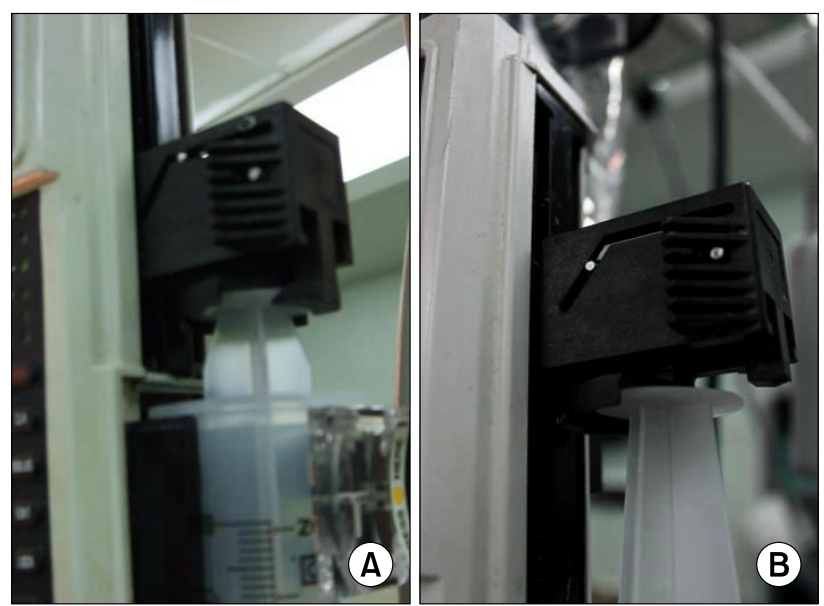

Fig. 1. A Baxter AS40A pump with a normal (A) and damaged (B) plunger clamp assembly which dose not firmly hold the plunger. twice. Surprisingly, the syringe loaded on AS40A was found to be empty and the missing $50 \mathrm{ml}$ of fentanyl was concluded to have been infused rapidly into the patient. However, even at this point there was no alarm signal or error message to indicate a dysfunctional plunger clamp.

Of many possible reasons, a siphon effect was strongly suspected to be the cause of this mishap, which refers to the continuous flow of liquids through enclosed conduit by the force of gravity once it had been initiated, because we can acknowledge that a complete spillage of $50 \mathrm{ml}$ syringe volume occurred within five minutes when the plunger lock was unclamped and manually started. Although high-dose fentanyl administration has been seen to be hemodynamically stable [1], such as in this case, bolus administration of another drug such as dopamine or norepinephrine, which is usually delivered using a Baxter infusion pump, may have catastrophic effects. Although a newer version of the Baxter AS40A pump has an alarm sensor for the syringe plunger lock, understanding the specifics of commonly used, older equipment in the operating room is crucial [2], and meticulous attention to a possibly improperly secured plunger head is always warranted in order to avoid potentially adverse outcomes.

\section{References}

1. Coda BA. Opiod. In: Barash PG, Cullen BF, Stoelting RK, eds. Clinical Anesthesia. 5th ed. Philadelphia, Lippincott Williams \& Wilkins. 2006, pp 353-83.

2. Christodoulou C. Incident with a Baxter AS40A syringe pump: vigilance is warranted. Can J Anaesth 2004; 51: 185-6.

Corresponding author: Sung-Hoon Kim, M.D., Department of Anesthesiology and Pain Medicine, University of Ulsan College of Medicine, Asan Medical Center, 388-1, Pungnap-dong, Songpa-gu, Seoul 138-736, Korea. Tel: 82-2-3010-3868, Fax: 82-2-3010-6790, E-mail: shkimans@amc.seoul.kr (c) This is an open-access article distributed under the terms of the Creative Commons Attribution Non-Commercial License (http:// creativecommons.org/licenses/by-nc/3.0/), which permits unrestricted non-commercial use, distribution, and reproduction in any medium, provided the original work is properly cited. 